\title{
Especies de Cryptosporidum en pacientes inmunodeficientes e inmunocompetentes de Valparaíso. Estudio descriptivo
}

\author{
Patricia Neira O., Nelson Muñoz S., Gonzalo Wilson L., \\ M. Elizabeth Barthel M., María J. Rosales L. y Carlos Henríquez R.
}

\section{Cryptosporidium species in immunodeficient and immunocompetent patients of Valparaíso. A descriptive study}

Objective: Genetical characterization of human Cryptosporidium isolates to determine species diversity. Patients and Methods: A cross-sectional study in Valparaiso, Chile, was performed. A total of 458 patients participated in the study: 259 immunodeficient (HIV, cancer, renal transplant hyper-IgM syndrome, HIV and unintended pregnancy) and 178 immunocompetent individuals provided stool samples and 21 patients bile samples. Results: We obtained $29(6.3 \%)$ positive samples. 25 (9.7\%) derived from immunodeficient patients: $18(7.3 \%)$ from HIV patients and 7 from patients with other immunodeficiencies. The remaining $4(2.2 \%)$ samples originated from immunocompetent individuals. Cryptosporidium genotyping was performed by nested polymerase chain reaction (PCR) and restriction fragments length polymorphism and/or PCR followed by sequencing of the SSU rRNA from oocysts in stool samples. 4 species were identified: C. parvum, C. hominis, C. muris, and C. meleagridis. In immunodeficient patients, 16 C. parvum, 8 C. hominis, and 1 C. muris strain were identified. In immunocompetent participants, 3 C. hominis and 1 C. meleagridis isolate were found. Conclusion: The results indicate that zoonotic and anthroponotic transmission occurs and that $C$. parvum is the predominant species in our study population. Cryptosporidium species of zoonotic transmission accounted for $62 \%$ of the human infections detected in this study.

Key words: Cryptosporidium parvum, C. hominis, C. meleagridis, C. muris, inmunocompetent, immunodeficient, descriptive study.

Palabras clave: Cryptosporidium parvum, C. hominis, C. meleagridis, C. muris, inmunocompetente, inmunodeficiente, estudio descriptivo.

\section{Introducción}

$\longrightarrow$ ryptosporidium spp son protozoos del phylum Apicomplexa, ubicuos en el ambiente, que ocasionan infección aguda y autolimitada. Del punto de vista clínico, las personas inmunocompetentes pueden ser asintomáticas o manifestar cuadros de diarrea que tienden a ser graves y persistentes, con un alto grado de morbilidad y mortalidad en los infectados inmunodeficientes.

Las especies de Cryptosporidium se han reportado en una amplia variedad de vertebrados ${ }^{1}$. A partir de ellos puede infectarse el hombre por diversos mecanismos de transmisión: contacto directo con personas (antroponótico) o animales (zoonótico) infectados, ingestión de alimentos y agua contaminada ${ }^{2}$. Existe evidencia de al menos 25 especies y 61 genotipos en diversos hospederos y con amplia distribución geográfica ${ }^{1,3}$. Las especies del parásito se han validado sobre la base de la morfología de los ooquistes, la biología del desarrollo, la especificidad del hospedero, el sitio de la infección, la histopatología y las diferencias genéticas establecidas mediante la biología molecular. También se utiliza el depósito en un museo de una muestra del parásito, como lo establece el Código Internacional para Nomenclatura Zoológica (ICZN por su sigla en inglés) $)^{3,4}$.

Los análisis genéticos revelan que al menos ocho especies (C. hominis, C. parvum, C. meleagridis, C. felis, C. canis, C. suis, C. muris y C. ubiquitum) y siete genotipos de Cryptosporidium (mono, similar a C. andersoni, ardilla, zorrillo, caballo, conejo y cerdo genotipo II) están asociados con enfermedad en seres humanos ${ }^{5-7}$, siendo $C$. parvum y C. hominis responsables de más de $90 \%$ de los casos humanos de criptosporidiosis ${ }^{8}$. Cabe destacar que estas especies se pueden distinguir solamente mediante técnicas moleculares ${ }^{9}$, debido a que la similitud de las características morfométricas de los ooquistes impide la diferenciación mediante tinción ácido alcohol resistente, como la de Ziehl-Neelsen. Esta última sigue siendo la más usada rutinariamente, debido a su bajo costo en relación con la mayor sensibilidad de las técnicas moleculares, para la búsqueda del protozoo en Chile.

Por otra parte, las infecciones con esas especies del
Universidad de Valparaíso, Chile.

Facultad de Medicina.

Departamento de Preclínicas Escuela de Medicina.

Cátedra de Parasitología (PNO, NMS)

Facultad de Ciencias,

Departamento de Estadística,

Centro de Estudios Estadísticos (CHR).

Hospital Carlos van Buren, Valparaíso, Chile.

Policlínico de Inmunología (GWL, MEB).

Universidad de Granada,

España.

Facultad de Ciencias,

Instituto de Biotecnología (MJRL)

Los autores declaran no tener conflicto de interés.

Financiamiento: Dirección de Investigación Universidad de Valparaíso (Proyecto DIPUV 01/2005).

Recibido: 25 de noviembre de 2010 Aceptado: 15 de agosto de 2011

Correspondencia a: Patricia Neira Otero patricia.neira@uv.cl 
protozoo no se presentan sólo en pacientes inmunodeficientes. En individuos inmunocompetentes se ha reportado la presencia de $C$. meleagridis ${ }^{10}, C$. canis $^{11}, C$. felis $^{9}$, C. suis ${ }^{12}$, C. muris ${ }^{13}$, así como los genotipos ciervo, conejo, mono y caballo ${ }^{14-17}$.

Para conocer cuáles son las especies que circulan en Valparaíso, Chile, y cómo se distribuyen según diferentes factores analizados, se genotipificó los ooquistes de Cryptosporidium procedentes de seres humanos de Valparaíso.

\section{Material y Método}

Se incluyeron 517 personas internadas o ambulatorias procedentes de la Región de Valparaíso, Chile. Del total de pacientes, 458 entregaron sus muestras biológicas para el estudio; 245 pacientes inmunodeficientes (con infección por VIH/SIDA) fueron referidos por los médicos responsables del policlínico de Inmunología del hospital Carlos van Buren; 14 pacientes inmunodeficientes no infectados por VIH: 6 oncológicos, 5 sometidos a trasplante de órganos, dos con síndrome de hiper IgM y una mujer embarazada, fueron referidos por los médicos especialistas tratantes. El estudio fue de corte transversal con un seguimiento longitudinal opcional en los casos positivos. El criterio de ingreso fue la voluntad de proporcionar al menos una muestra de deposiciones y responder una encuesta con preguntas de antecedentes personales y epidemiológicos. Los antecedentes clínicos de los pacientes con infección por VIH/SIDA (CD4, carga viral) fueron obtenidos por los médicos desde la ficha clínica.

Las muestras de 178 pacientes inmunocompetentes procedieron de 78 personas derivadas desde la consulta médica al Laboratorio de Parasitología de la Escuela de Medicina de la Universidad de Valparaíso, con solicitud de examen coproparasitario para diagnóstico de enteroparásitos; control parasitario de 27 niños procedentes de la Corporación de Nutrición Infantil (CONIN); 58 niños y adolescentes del archipiélago de Juan Fernández, cuyos padres se interesaron en participar del estudio, y el examen de control de 15 adultos que atienden los animales cautivos en el zoológico de Quilpué. Además, se estudiaron 21 muestras de bilis obtenidas mediante sondeo duodenal de pacientes adultos ambulatorios inmunocompetentes.

El protocolo del estudio fue aprobado por el Comité de Ética de la Facultad de Medicina de la Universidad de Valparaíso. Todos los pacientes dieron su consentimiento informado para participar en el estudio proporcionando sus muestras biológicas para los análisis.

Todas las muestras de deposiciones fueron analizadas mediante la técnica de Telemann modificada. La búsqueda de ooquistes de Cryptosporidium se efectuó en 21 muestras de bilis y 437 muestras frescas de deposiciones con tinción de Ziehl-Neelsen, de pacientes con y sin diarrea, post concentración mediante centrifugación (5.000 rpm por $10 \mathrm{~min}$ ). Se consideró como muestra positiva aquella que presentó uno o más ooquistes de Cryptosporidium sp identificados de acuerdo a las características morfométricas y de tinción.

Los ooquistes aislados de cada paciente fueron sometidos a un proceso de congelación y descongelación para la ruptura y la liberación de los esporozoítos y luego, mantenidos a $4^{\circ} \mathrm{C}$. Para la obtención del ADN genómico de Cryptosporidium se implementó un protocolo de extracción a partir de ooquistes en $200 \mu 1$ de materia fecal, de acuerdo a lo descrito en otro estudio ${ }^{18}$. El extracto resultante se almacenó a $-20^{\circ} \mathrm{C}$ hasta su uso.

La amplificación mediante RPC anidada de la SSU de los genes ADNr de Cryptosporidium, se realizó de acuerdo a dos protocolos: según lo descrito por Xiao y cols (1999 a y b) $)^{19-20}$ en $50 \mu 1$ de mezcla de reacción que contenía ADN de Cryptosporidium sin cuantificar; y el segundo protocolo, de acuerdo a lo publicado por Tiangtip y cols $2002^{21}$, en $25 \mu 1$ de mezcla de reacción que contenía ADN de Cryptosporidium cuantificado mediante espectrofotometría.

El primer protocolo se continuó con el polimorfismo de la longitud de los fragmentos de restricción (PLFR) del producto de la segunda RPC utilizando las enzimas de restricción SspI y AseI. Las bandas obtenidas se usaron para la diferenciación de especies y genotipos, de acuerdo a lo previamente descrito ${ }^{22}$. El segundo protocolo se continuó con la purificación y posterior secuenciación bidireccional del producto de la RPC anidada en cuatro alícuotas de ADN de cada muestra, de acuerdo a lo descrito previamente ${ }^{18}$. En ambos protocolos se usó como control positivo ooquistes de C. parvum aislados de ternero de la granja Puleva, Granada, España y de C. parvum y C. hominis, aislados y caracterizados previamente de pacientes con infección por VIH de Chile; además se usó un control negativo ${ }^{22}$.

Las secuencias bidireccionales de cada producto de RPC fueron alineadas y editadas para comparación con su electroferograma. Posteriormente, se alinearon y compararon con lo reportado internacionalmente para obtener la secuencia de consenso final. La secuencia final se utilizó para buscar en la base de datos Genbank las similitudes con el Centro Nacional para Información de Biotecnología (NCBI, por su sigla en inglés), a través de la herramienta BlastN (http://blast.ncbi.nlm.nih.gov/Blast). Las secuencias parciales obtenidas fueron depositadas en la base de datos Genbank (E.U.A.), EMBL (Europa) y DDBJ (Japón).

De los pacientes en quienes se dispuso de una muestra de deposición positiva se realizó un estudio descriptivo cuyas variables de interés primario, para los pacientes inmunodeficientes no infectados por VIH e inmunocompetentes, fueron sexo, edad, diarrea y especie de 
Cryptosporidium. En los pacientes con infección por VIH/SIDA, además se consideró el recuento de CD4 $\left(\right.$ céls $\left./ \mathrm{mm}^{3}\right)$. Estas variables primarias fueron relacionadas con lugar de residencia, consumo de alimentos crudos (vegetales, mariscos y pescados), presencia de mascotas (perros, gatos y/o aves) en el hogar. Además, dependiendo de la presencia o ausencia de Cryptosporidium, se efectuó comparaciones entre grupos (por sexo, edad, análisis de variación estacional de la aparición de las infecciones). Para ello, se obtuvo probabilidades de significación del tipo descriptiva, ya que, las muestras de pacientes no fueron seleccionadas al azar. La base de datos fue administrada y analizada utilizando el software estadístico Stata versión 11.2.

\section{Resultados}

De un total de 517 personas, se obtuvieron 458 muestras biológicas (437 de deposiciones y 21 de bilis), distribuidas según sexo y promedio de edad como se muestra en la Tabla 1. Se obtuvieron 29 muestras de deposiciones positivas mediante Ziehl Neelsen para Cryptosporidium spp (6,3\%); 24 pacientes eran de sexo masculino; 26 y 3 en pacientes con y sin diarrea, respectivamente. Veinticinco de 259 muestras positivas fueron de pacientes inmunodeficientes $(9,7 \%)$ : 18 (7,5\%) con infección por VIH/SIDA. Los 7 restantes correspondieron a 4 pacientes oncológicos, uno sometido a trasplante renal, un síndrome de hiper-IgM y una mujer embarazada. Cuatro de 178 $(2,2 \%)$ muestras positivas fueron de pacientes inmunocompetentes con diarrea, uno con síndrome de Down. No se observó el protozoo en las muestras de bilis.

Mediante las herramientas moleculares (RPC-PLFR o RPC-secuenciación), se identificó en pacientes inmunodeficientes con VIH/SIDA: 9 C. parvum, 8 C. hominis y un C. muris, con 91 y 100\% de identidad a lo publicado. En los otros pacientes inmunodeficientes no infectados por VIH, sólo se identificó C. parvum con 95\% de identidad. En pacientes inmunocompetentes se identificó tres $C$. hominis y un C. meleagridis en un paciente con síndrome de Down, con 91 y 98\% de identidad con lo publicado en las bases de datos GenBank/EMBL/DJBJ. La distribución de especies de Cryptosporidiurn por sexo, en pacientes inmunodeficientes e immunocompetentes se muestra en la Tabla 2. Sólo C. parvum se presentó en pacientes sin diarrea o portadores asintomáticos (dos en pacientes con infección por VIH y uno en síndrome de hiper IgM). Se detectó variabilidad intra-específica en los aislados de $C$. hominis y C. parvum.

El recuento promedio de CD4, en los pacientes con infección por VIH/SIDA y positivos para Cryptosporidium fue de 99,4 céls $/ \mathrm{mm}^{3}$. CD4 de 13 céls $/ \mathrm{mm}^{3}$ se detectó en el paciente infectado por VIH con C. muris, y un promedio de 71,1 y 134,2 céls/ $/ \mathrm{mm}^{3}$ en los pacientes positivos para C. hominis y C. parvum, respectivamente. La distribución de especies de Cryptosporidium en relación al recuento de CD4 (céls $/ \mathrm{mm}^{3}$ ) se muestra en la Figura 1. No se observó asociación entre carga viral (copias ARN/ml) y especie de Cryptosporidium (datos no mostrados). Sólo seis pacientes se encontraban en terapia anti-retroviral (TARV) en el momento del diagnóstico de criptosporidiosis; cuatro presentaron C. parvum y dos C. hominis.

En el análisis de procedencia de los pacientes, de los 29 infectados, nueve casos residían en el sector de Playa Ancha y cinco casos en el centro de Valparaíso; los 15 restantes en diversos cerros de Valparaíso, sin concentrarse en alguno en particular.

De los 245 pacientes con infección por VIH/SIDA, 210 respondieron al consumo de alimentos crudos; de los cuales 197 (80,4\%) reconocieron consumir verduras y frutas crudas, pero sólo $32(13,1 \%)$ pescados y mariscos crudos. Con relación a la presencia de mascotas en el domicilio, $45(18,4 \%)$ tenían perro (entre uno y cinco animales/hogar) y $21(8,6 \%)$ tenían gato (entre uno y cuatro animales/hogar); al respecto, la gran mayoría no efectuaba control veterinario en sus mascotas. La minoría de los pacientes refirió no poseer agua potable ni alcantarillado en su vivienda; el agua era obtenida de pozo o vertiente y el pozo negro constituía el sistema de eliminación de excretas, en estos casos. Los resultados se resumen en la Tabla 3.

Entre los antecedentes epidemiológicos de los pacientes inmunodeficientes no infectados por VIH, la mujer embarazada y dos pacientes sometidos a trasplante renal señalaron que consumían verduras y frutas crudas, no así pescados y mariscos. La mujer embarazada poseía cuatro perros adultos y tres gatos: uno adulto y dos cachorros. Los pacientes que recibieron trasplante indicaron que tenían un perro adulto cada cual; uno no poseía agua potable ni alcantarillado en su vivienda y la mujer embarazada obtenía el agua desde un pozo. Se demostró la transmisión zoonótica de C. parvum a partir de gatos cachorros mascotas en la paciente embarazada ${ }^{23}$.

La relación entre presencia de Cryptosporidium spp y la época del año se muestra en la Tabla 4.

Las secuencias parciales obtenidas fueron depositadas en las bases de datos de Genbank/EMBL/DDBJ bajo los No de acceso: GQ368454-GQ368457, GQ865520GQ865526, GU055299-GU055303, GQ355890 у GQ355895.

En los pacientes con infección por VIH/SIDA, se detectó la presencia de otros enteroparásitos, entre los que se incluyen: Giardia duodenalis (n: 4), Entamoeba histolytica, (n: 3) Isospora belli (n: 2), Strongyloides stercoralis (n: 2) e Hymenolepis nana (n: 2). Sólo en un paciente con infección por VIH se detectó co-infección entre S. stercoralis y C. parvum. Además, se observó la 
Tabla 1. Distribución por sexo y promedio de edad de pacientes inmunodeficientes e inmunocompetentes con criptosposidiosis* ( $\mathrm{n}=437$ )

Estado inmunitario

Inmunodeficiente
Infección por VIH/SIDA
Oncológico
Trasplante renal
Síndrome de hiper IgM
Embarazo

Sexo y edad (años)

M \%*

$\begin{array}{rl}207 & 84,5 \\ 4 & 4 / 6^{*} \\ 4 & 4 / 5^{*} \\ 2 & 2 / 2^{*}\end{array}$

Edad
promedio

(años)
(Rango)

(R)

\section{F}

$\begin{array}{rr}36,3 & (10-71) \\ 7,3 & (4-10) \\ 46,0 & (44-48) \\ 2,6 & (0,3-5)\end{array}$

Inmunocompetente

Total

$86 \quad 19,7$

303

69,3

$\begin{array}{ll}16,9 & (0,1-69) \\ 69,3 & (0,1-71)\end{array}$

$(0,1-69)$
$(0,1-71)$

69,3

134

$\begin{array}{rcc}38 & 15,5 & 36,5 \\ 2 & 2 / 6^{*} & 9 \\ 1 & 1 / 5^{*} & 59 \\ 1 & & \\ & 1 / 1 * & 28\end{array}$

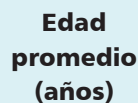

(años)

*No se obtiene porcentaje por escaso número de muestras.

\section{Tabla 2. Distribución de especies de Cryptosporidium por sexo, en pacientes inmunodeficientes e immunocompetentes de Valparaíso, Chile}

\begin{tabular}{lcccccc} 
Especie de & \multicolumn{3}{c}{ Infección por VIH/SIDA } & \multicolumn{3}{c}{ Inmunodeficientes no SIDA** } \\
Cryptosporidium & $\mathbf{M}$ & $\mathbf{F}$ & $\mathbf{T}$ & $\mathbf{M}$ & $\mathbf{F}$ & $\mathbf{T}$ \\
& $\mathbf{2 0 7}$ & $\mathbf{3 8}$ & $\mathbf{2 4 5}$ & $\mathbf{1 0}$ & $\mathbf{4}$ & $\mathbf{1 4}$ \\
C. parvum & 9 & 0 & 9 & 5 & 2 & 7 \\
C. horninis & 6 & 2 & 8 & 0 & 0 & 0 \\
C. meleagridis & 0 & 0 & 0 & 0 & 0 & 0 \\
C. muris & 1 & 0 & 1 & 0 & 0 & 0 \\
Total & 16 & 2 & 18 & 5 & 2 & 7
\end{tabular}

\begin{tabular}{cccccc}
\multicolumn{2}{c}{ Inmunocompetentes } & \multicolumn{3}{c}{ Total* $^{*}$} \\
$\mathbf{M}$ & $\mathbf{F}$ & $\mathbf{T}$ & $\mathbf{M}$ & $\mathbf{F}$ & $\mathbf{T}$ \\
$\mathbf{8 6}$ & $\mathbf{9 2}$ & $\mathbf{1 7 8}$ & $\mathbf{3 0 3}$ & $\mathbf{1 3 4}$ & $\mathbf{4 3 7}$ \\
0 & 0 & 0 & 14 & 2 & 16 \\
2 & 1 & 3 & 8 & 3 & 11 \\
1 & 0 & 1 & 1 & 0 & 1 \\
0 & 0 & 0 & 1 & 0 & 1 \\
3 & 1 & 4 & 24 & 5 & 29
\end{tabular}

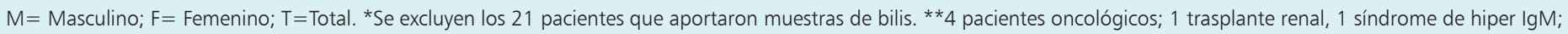
1 mujer embarazada.

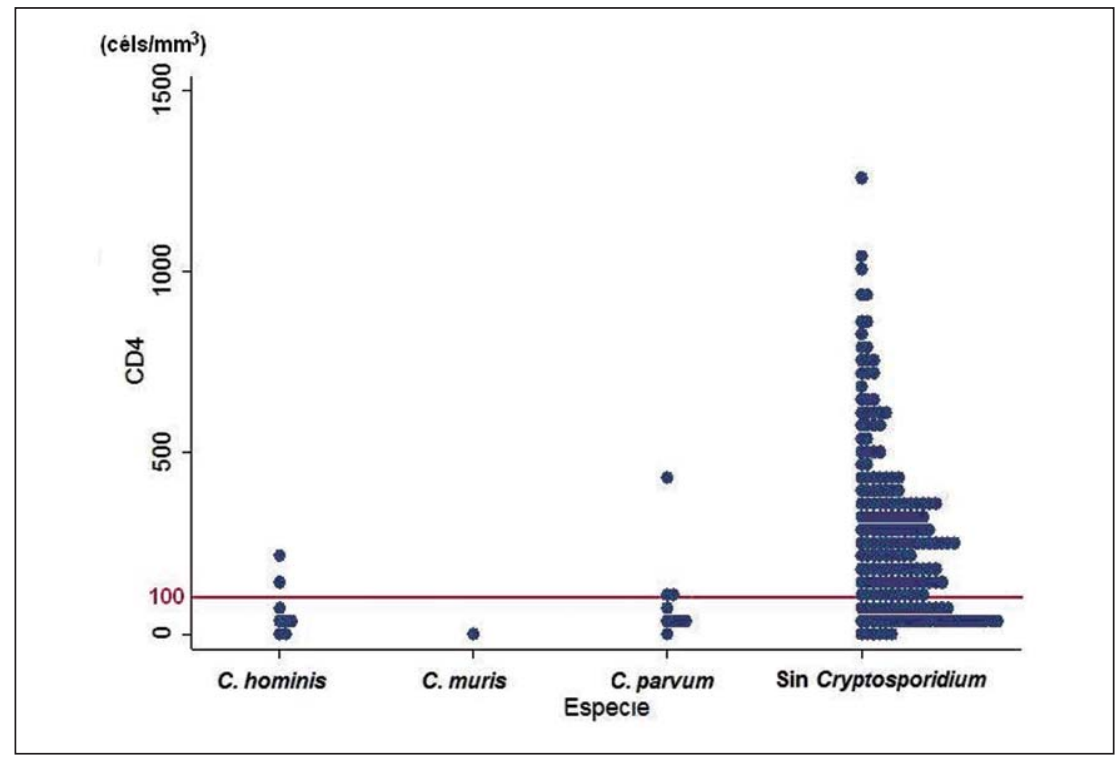

Figura 1. Recuento de CD4 (céls/ $\mathrm{mm}^{3}$ ) de acuerdo a la especie de Cryptosporidium detectada. presencia de especies comensales: Entamoeba coli (n: 14) y Endolimax nana (n: 6). Entre los inmunodeficientes no infectados por VIH, sólo un paciente oncológico presentó huevos de $H$. nana. Otros elementos detectados en pacientes inmunocompetentes fueron: G. duodenalis, Trichuris trichiura, Diphyllobothrium latum, Dipylidium caninum y Blastocystis hominis, uno en cada caso

La base de datos de personas procedentes del archipiélago de Juan Fernández, del zoológico de Quilpué, CONIN y aquellas que proporcionaron las muestras de bilis, no se analizaron mediante el software estadístico Stata versión 11, debido a que los resultados obtenidos fueron negativos para Cryptosporidium spp. Todas las muestras de bilis y las procedentes de CONIN y el zoológico de Quilpué fueron negativas para otros enteroparásitos; en el zoológico sólo una persona presentó infección por E. nana (protozoo comensal). En las 58 muestras procedentes del archipiélago de Juan Fernández se detectaron la presencia de G. duodenalis (n: 4), B. hominis (n: 6), E. coli (n: 2) y E. nana;(n: 2); 45 muestras resultaron negativas. 


\begin{tabular}{|c|c|c|c|c|c|c|c|c|c|c|c|c|c|c|c|c|c|c|}
\hline \multirow{3}{*}{ Cryptosporidium spp } & \multicolumn{6}{|c|}{ Consumo de alimentos crudos } & \multicolumn{6}{|c|}{ Presencia de mascota en el hogar } & \multicolumn{6}{|c|}{ Disposición en el hogar } \\
\hline & \multicolumn{3}{|c|}{ Verduras y frutas } & \multicolumn{3}{|c|}{$\begin{array}{c}\text { Pescados y } \\
\text { mariscos }\end{array}$} & \multicolumn{3}{|c|}{ Perro } & \multicolumn{3}{|c|}{ Gato } & \multicolumn{3}{|c|}{ Agua } & \multicolumn{3}{|c|}{ Alcantarillado } \\
\hline & $(+)$ & $(-)$ & Total & $(+)$ & $(-)$ & Total & $(+)$ & $(-)$ & Total & $(+)$ & $(-)$ & Total & $(+)$ & $(-)$ & Total & $(+)$ & $(-)$ & Total \\
\hline Ausente & 183 & 14 & 197 & 31 & 166 & 197 & 50 & 177 & 227 & 24 & 203 & 227 & 196 & 4 & 200 & 192 & 8 & 200 \\
\hline Presente & 11 & 1 & 12 & 1 & 12 & 3 & 3 & 15 & 18 & 1 & 17 & 18 & 11 & 1 & 12 & 10 & 2 & 12 \\
\hline Total & 194 & 15 & 209 & 32 & 178 & 210 & 53 & 192 & 245 & 25 & 220 & 245 & 207 & 5 & 212 & 202 & 10 & 212 \\
\hline OR ( $p$ value) & \multicolumn{3}{|c|}{$0,84(0,873)$} & \multicolumn{3}{|c|}{$0,45(0,446)$} & \multicolumn{3}{|c|}{$0,708(0,597)$} & \multicolumn{3}{|c|}{$0,49(0,507)$} & \multicolumn{3}{|c|}{$0,224(0,198)$} & \multicolumn{3}{|c|}{$0,208(0,066)$} \\
\hline
\end{tabular}

\section{Discusión}

Aunque el género Cryptosporidium se diagnostica por medio de la visualización de ooquistes teñidos con Ziehl-Neelsen mediante microscopia de luz, las técnicas de biología molecular (RPC anidada asociada a PLFR y/o RPC y la posterior secuenciación), permiten el diagnóstico de certeza de la especie involucrada en la infección; son útiles para el diagnóstico de infecciones leves y para constatar la eliminación del parásito post TARV en los pacientes con infección por VIH/SIDA.

El estudio permitió la identificación en seres humanos de cuatro especies de Cryptosporidium circulantes en el territorio: C. parvum, C. hominis, C. meleagridis y C. muris. Esto demostró la transmisión zoonótica y antroponótica del protozoo en la Región de Valparaíso. De las 29 muestras positivas, los 11 casos de C. hominis corresponden a transmisión antroponótica. Puesto que la transmisión de C. parvum (18/29 casos en este estudio) puede ser antroponótica o zoonótica, la genotipificación de las infecciones detectadas que afecta a la población humana de Valparaíso (Tabla 2), no permite demostrar la predominancia de transmisión por uno u otro mecanismo. La presencia de un C. muris y un C. meleagridis corresponde a transmisión zoonótica.

Al analizar la distribución de las infecciones por Cryptosporidium spp de acuerdo a diversos factores epidemiológicos (Tablas 3 y 4), el análisis estadístico no demostró significancia. Estos resultados (factores analizados y estudio estadístico) podrían variar si se usan técnicas de búsqueda más sensibles que la tinción de Ziehl-Neelsen, lo que aumentaría la casuística. Resulta interesante anotar que al preguntar por ingesta de pescado y mariscos crudos, muchas personas no consideran el cebiche como alimento crudo.

El análisis de los antecedentes clínicos de los pacientes con SIDA permite efectuar los siguientes comentarios: en aquellos pacientes con diarrea, la presencia del parásito se acompañó con recuento promedio de CD4 de 99,4 céls $/ \mathrm{mm}^{3}$. Este resultado fue superior al obtenido en los
Tabla 4. Distribución estacional de especies de Cryptosporidium en pacientes inmunodeficientes e inmunocompetentes de Valparaíso

\begin{tabular}{|lccc|}
\hline Cryptosporidium spp & \multicolumn{2}{c|}{ Estación de diagnóstico } & Total \\
& Primavera - verano & Otoño - invierno & \\
Ausente & 110 & 124 & 234 \\
Presente & 9 & 16 & 25 \\
Total & 119 & 140 & 259 \\
OR $=1,58$ (p-value: 0,297$)$ & & & \\
\hline
\end{tabular}

primeros pacientes con SIDA $\left(71,7\right.$ céls $\left./ \mathrm{mm}^{3}\right)$ y con criptosporidiosis en $\mathrm{Chile}^{24}$, en la era previa a la introducción de TARV. Cryptosporidium spp no se detectó en aquellos casos con diarrea cuyo recuento de CD4 fue igual o superior a 200 céls $/ \mathrm{mm}^{3}$ (Figura 1). En estos pacientes, la frecuencia de infección $(7,5 \%)$ por este parásito, podría explicarse por un inadecuado nivel plasmático de los farmacos anti-retrovirales, como resultado de un aumento del metabolismo o excreción de los fármacos, debido a la distribución alterada dentro de los distintos compartimientos del cuerpo, o a una tardía concentración máxima en el plasma ${ }^{25}$; también por la no adhesión de los pacientes a la terapia.

En la etapificación de los pacientes con infección por VIH/SIDA, la categoría clínica $\mathrm{C}$ es la etapa indicadora de SIDA; siendo la criptospordiosis de más de un mes de duración, uno de los cuadros definitorios de esta fase. La diferencia en el recuento promedio de CD4 entre pacientes asintomáticos $\left(287,9\right.$ céls $\left./ \mathrm{mm}^{3}\right)$ y sintomáticos $\left(99,4\right.$ céls $\left./ \mathrm{mm}^{3}\right)$ fue menor en este estudio que en uno efectuado en E.U.A., donde el recuento promedio fue de 312 y 57 céls $/ \mathrm{mm}^{3}$, respectivamente ${ }^{26}$. En Uganda, los pacientes con criptosporidiosis tienen en promedio un recuento de CD4 muy inferior, que alcanza a 16 céls/ $\mathrm{mm}^{3}$, similar a lo detectado en el paciente con C. muris de este estudio; en India, el recuento promedio de CD4 en pacientes con infección por VIH con diarrea varía de 
$145^{28}$ a 226 céls $/ \mathrm{mm}^{329}$. Actualmente, la TARV ha hecho una gran diferencia en el pronóstico de estos pacientes. El reconocer a una persona con infección por VIH en etapa temprana, permite poder intervenir en la diseminación de la infección y al paciente se le puede ofrecer un tratamiento que le permitirá mejorar su calidad de vida, mientras cumpla cabalmente con el tratamiento y sus controles. Ello se evidencia en los resultados de este estudio, donde los pacientes que no adhirieron a TARV y presentaban Cryptosporidium, han fallecido.

A pesar de la mayor frecuencia de infecciones $(58,6 \%)$ en los meses de otoño e invierno (17/29 casos), el análisis estadístico descriptivo de la relación entre presencia de Cryptosporidium spp y la época del año no resultó significativa (Tabla 4). Sería importante establecer si los periodos de otoño e invierno alargan la viabilidad de los ooquistes ${ }^{30}$ y potencian las infecciones en los diversos hospederos.

Si bien se observó una dispersión en la procedencia de los pacientes, el haber detectado de los 29 positivos, 9 casos en Playa Ancha y 5 en el centro de Valparaíso (zona suburbana y urbana, respectivamente), haría suponer un factor de riesgo de mayor importancia, por la probable mayor contaminación de esos ambientes con el protozoo.

Es lógico pensar que los pacientes inmunodeficientes, especialmente aquellos con SIDA, podrían ser susceptibles a infecciones oportunistas con una amplia variedad de especies y genotipos del protozoo. La distribución de C. parvum y C. hominis en humanos difiere según las regiones geográficas; $C$. hominis es más prevalente (62\%) en algunos países de América del Norte y del Sur, Australia y África; en países europeos ambas especies son comunes en seres humanos ${ }^{8}$. En el Medio Oriente, predomina $C$. parvum y en el resto del mundo, especialmente en los países en vías de desarrollo, C. hominis es, por lo general, la especie más frecuente en los seres humanos ${ }^{8}$. También se ha demostrado, en E.U.A. e Irlanda que la proporción de infección por $C$. parvum es mayor que $C$. hominis en áreas rurales que urbanas ${ }^{5,31-32}$.

En relación con el tipo de inmunocompromiso, los pacientes con infección por VIH/SIDA se infectan mayoritariamente con C. hominis en E.U.A., Perú, Brasil, Kenia, Malawi, Vietnam e India ${ }^{28,33-34}$ y con C. parvum en el Reino Unido ${ }^{35}$. Estos datos sugieren que los pacientes infectados con VIH tienen mayor riesgo de infección con C. hominis y C. parvum como sucedió en este estudio donde sólo en un caso se detectó $C$. muris. En algunas zonas geográficas parecen ser más propensos a otras especies de origen animal, tales como: C. andersoni, C. canis, C. felis, C. muris, C. wrairi, C. meleagridis, C. baileyi, C. saurophylum y C. serpentis ${ }^{8}$, lo que sugiere que la transmisión zoonótica juega un papel importante en pacientes inmunodeficientes ${ }^{36}$; sin embargo, aunque las especies identificadas en humanos pueden ser ge- néticamente similares a las de los animales, existe poca evidencia de que se produzcan infecciones cruzadas ${ }^{36}$. Se pensaba que estas especies sólo infectaban a pacientes inmunodeficientes y actualmente se sabe que también infectan a individuos inmunocompetentes ${ }^{8,9}$.

En pacientes oncológicos, la criptosporidiosis puede constituir una enfermedad grave y debilitante en personas con leucemia y otras neoplasias hematológicas ${ }^{37}$, en $2 / 6$ de los pacientes de este estudio el diagnóstico de base correspondía a leucemia, lo que pudiera ser un factor determinante en el desenlace fatal al no existir terapia específica, como sucedió con el primer caso de criptosporidiosis detectado en Chile ${ }^{38}$.

En pacientes sometidos a trasplantes, C. parvum ocasiona diarrea $^{39}$, infecciones asintomáticas u oligosintomáticas $^{40}$. Se ha aislado en 34,8 y 18,8\% de los pacientes receptores de trasplante renal en Brasil $^{41}$ y Turquía ${ }^{42}$, respectivamente. El único paciente que recibiera un trasplante renal de este estudio presentó diarrea por C. parvum; su criptosporidiosis pudiera corresponder a una reinfección o al empeoramiento de una infección leve debido al uso prolongado de fármacos inmunosupresores, como se ha observado en experimentos con animales ${ }^{43-44}$; o bien a una infección actual. Estos pacientes constituyen un grupo de riesgo para patógenos oportunistas y deberían periódicamente someterse a controles copro-parasitarios para búsqueda de Cryptosporidium $\mathrm{sp}^{39}$, aparte de manejar medidas de prevención de infecciones transmitidas por fecalismo ambiental.

Existen algunas publicaciones de diarrea crónica asociada con Cryptosporidium spp en pacientes con inmunodeficiencias primarias ${ }^{45,46}$ en los que se refleja el escaso número de casos disponibles para su estudio. El informe anual del grupo de inmunodeficiencias primarias de Chile (CHILEGID), reportaba ocho chilenos de los 34 pacientes latinoamericanos, hasta el año 2004. En este estudio, el haber conservado las muestras fecales en formol salino al 5\%, desde 1987, permitió comprobar la presencia de $C$. parvum en un paciente con síndrome de hiper IgM. En los pacientes con inmunodeficiencias primarias, la infección por Cryptosporidium spp puede asociarse con colangitis esclerosante con progresión a cirrosis e insuficiencia hepática ${ }^{47}$, lo que debe tenerse presente frente a futuros nuevos casos.

Con relación a criptosporidiosis y embarazo, en la gestante primípara se demostró, identificó molecularmente y comprobó la transmisión zoonótica de $C$. parvum a partir de sus cachorros de gatos ${ }^{23}$, constituyendo el primer caso en Chile de transmisión entre vertebrados (humano y mascota) que co-habitan. El riesgo potencial durante el embarazo, a pesar de la normalidad de la mayoría de las funciones inmunitarias, probablemente se debería a la disminución en la inmunidad mediada por células para determinados antígenos. Sin embargo, se desconoce, si 
durante el embarazo estas células son suficientes para controlar la evolución autolimitada de la criptosporidiosis ${ }^{48}$, como sucede en personas inmunocompetentes.

El haber detectado Cryptosporidium spp en 2,2\% de los pacientes inmunocompetentes con diarrea, permite comprobar el descenso en la prevalencia de este protozoo en relación a estudios previos ${ }^{49}$. Los casos se presentaron en niños entre 2 y 14 años y un adulto joven de 30 años. En los niños y en especial en aquellos con defectos genéticos, la presencia del parásito se explicaría por las inadecuadas conductas higiénicas propias de la edad las que habrían permitido la infección en el paciente con síndrome de Down. En el adulto, por el hecho de que la parasitosis está presente en el medio ambiente y por no haberse encontrado en adultos inmunocompetentes con anterioridad, se recomendó al paciente, descartar infección por VIH, estudio que resultó negativo.

Las especies detectadas en pacientes inmunocompetentes fueron C. hominis y C. meleagridis. Cryptosporidium meleagridis se describió originalmente en el tracto respiratorio de pavos $^{9}$ y es la única especie que infecta naturalmente a aves y mamíferos. Actualmente se reconoce como un patógeno emergente en humanos ${ }^{10,50-51}$, se ha presentado en personas inmunocompetentes del Reino Unido ${ }^{52}$; es responsable de casi $1 \%$ de todas las infecciones en Inglaterra ${ }^{12}$ y de aproximadamente $10 \%$ de las producidas en Perú ${ }^{53}$, donde su prevalencia es tan alta como la de C. parvum ${ }^{54}$. En este estudio, C. meleagridis se detectó en un paciente con síndrome de Down; sin embargo, la fuente aviar no pudo ser reconocida.

La ausencia de Cryptosporidium spp, en el resto de los participantes de este estudio (procedentes de CONIN, el archipiélago de Juan Fernández y el zoológico de Quilpué), confirmaría que esta parasitosis es infrecuente en individuos inmunocompetentes sin síntomas digestivos; a lo que se suma las mejoras en el saneamiento básico que han permitido el descenso de las enteroparasitosis en general ${ }^{55}$. Los hallazgos parasitarios en personas procedentes del archipiélago de Juan Fernández estarían principalmente relacionados con el grado de cultura higiénica de la población estudiada, en especial en el grupo del sexo masculino.

Debido a que los parques zoológicos son lugares de concentración de animales, constituyen un riesgo potencial de diseminación de enfermedades zoonóticas; los resultados permiten extrapolar que durante este estudio descriptivo, existió riesgo de transmisión zoonótica de C. parvum a partir de mamíferos y de $C$. parvum y $C$. meleagridis a partir de aves (datos no mostrados). Sin embargo, el no haber detectado el parásito en humanos, permite deducir que en este parque zoológico se cumplen adecuadamente las normas de bioseguridad establecidas para las prácticas de manejo de los animales cautivos.

El sondeo duodenal, usado rutinariamente en la práctica médica en el estudio de diversas patologías, sólo permite el diagnóstico casual de infecciones parasitarias. La ausencia del parásito en los pacientes estudiados y la invasividad del método para la recolección del líquido duodenal, no justificaría su uso primario para fines diagnósticos de Cryptosporidium; sobre todo considerando que el método de detección para criptosporidiosis (ZiehlNeelsen) usado en este estudio es de baja sensibilidad.

\section{Conclusiones}

Aunque el género Cryptosporidium se diagnostica por medio de la visualización de ooquistes teñidos con Ziehl-Neelsen mediante microscopia de luz, las técnicas de biología molecular (RPC anidada asociada a PLFR y/o RPC y la posterior secuenciación), permiten el diagnóstico de certeza de la especie involucrada en la infección. Se demostró la utilidad para la determinación de fuentes y modos de transmisión del parásito, como sucedió con la mujer embarazada y sus cachorros de gatos. Este estudio descriptivo permitió la identificación mediante biología molecular de cuatro especies del protozoo en la Región de Valparaíso: C. parvum, C. hominis, C. muris y $C$. meleagridis. Se requiere mayor número de pacientes para poder establecer el comportamiento de esta infección en relación con las variables analizadas.

Agradecimientos: Nuestros sinceros agradecimientos a los pacientes de este estudio; al $\mathrm{PhD}$ en Biología Molecular Mario Párraga S. y a la bióloga Sra. Victoria Devia V. por la asesoría en las técnicas moleculares y al Sr. Ivo Carrasco W. por la secuenciación de las muestras.

\section{Resumen}

Objetivo: Caracterizar genéticamente Cryptosporidium spp para determinar la diversidad de especies en seres humanos. Pacientes y Métodos: estudio transversal realizado en Valparaíso, Chile, Un total de 458 pacientes participaron del estudio; 259 inmunodeficientes (pacientes con infección por VIH, oncológicos, con trasplante renal, síndrome de hiper IgM y una mujer embarazada sin infección por VIH) y 178 inmunocompetentes proporcionaron muestras fecales y 21 muestras de bilis. Resultados: Se obtuvieron 29 (6,3\%) muestras positivas; 25 (9,7\%) de inmunodeficientes: $18(7,3 \%)$ de pacientes con infección por VIH y 7 con otras inmunodeficiencias; los restantes $4(2,2 \%)$ fueron de personas inmunocompetentes. La genotipificación de Cryptosporidium se efectuó mediante reacción de polimerasa en cadena (RPC) anidada y el polimorfismo de la longitud de los fragmentos de restricción y/o RPC - secuenciación de la SSU ARNr, a partir de 
ooquistes en la muestra fecal. Se identificaron 4 especies: C. parvum, C. hominis, C. muris y C. meleagridis. En pacientes inmunodeficientes, se caracterizaron $16 \mathrm{C}$. parvum, $8 \mathrm{C}$. hominis y un $C$. muris; en inmunocompetentes: 3 C. hominis y un C. meleagridis. Conclusión: Los resultados indican que se produce transmisión zoonótica y antroponótica y que $C$. parvum es la especie predominante en este estudio. Las especies de Cryptosporidium de transmisión zoonótica representan el $62 \%$ en los seres humanos participantes de este estudio.

\section{Referencias}

1.- Xiao L, Ryan U M. Molecular Epidemiology. Fayer R, Xiao L (ed.), Cryptosporidium and cryptosporidiosis, 2nd ed. CRC Press, Boca Raton, FL; 2008, p. 119-72.

2.- Smith A T, Nichols R A B. Cryptosporidium: detección en el agua y los alimentos. Exp Méx 2010; 124: 61-79.

3.- Plutzer J, Karanis P. Genetic polymorphism in Cryptosporidium: an update. Vet Parasitol 2009; 165: 187-99.

4.- Fayer R, Santín M. Cryptosporidium xiaoi n. sp. (Apicomplexa: Cryptosporidiidae) in sheep (Ovis aries). Vet Parasitol 2009; 164: 192-200.

5.- Cacciò S M, Thompson R C, McLauchlin J, Smith H V. Unravelling Cryptosporidium and Giardia epidemiology. Trends Parasitol 2005; 21: 430-7.

6.- Smith H V, Cacciò S M, Tait A, McLauchlin J, Thompson R C. Tools for investigating the environmental transmission of Cryptosporidium and Giardia infections in humans. Trends Parasitol 2006; 22: 160-7.

7.- Kváč M, Kvetonová BS, Sak B, Ditrich O. Cryptosporidium pig genotype II in immunocompetent man. Emerg Infect Dis 2009; 15: 982-3.

8.- Xiao L. Molecular epidemiology of cryptosporidiosis: an update. Exp Parasitol 2010; 124: 80-9.

9.- $\quad$ Xiao L, Fayer R, Ryan U, Upton S J. Cryptosporidium taxonomy: recent advances and implications for public health. Clin Microbiol Rev 2004; 17: 72-97.

10.- Pedraza-Díaz S, Amar C F, McLauchlin J, Nichols G L, Cotton K M, Godwin P, et al. Cryptosporidium meleagridis from humans: molecular analysis and description of affected patients. J Infect 2001; 42: 243-50.

11.- Xiao L, Cama V A, Cabrera L, Ortega Y, Pearson J, Gilman R H. Possible transmission of Cryptosporidium canis among children and a dog in a household. J Clin Microbiol 2007; 45: 2014-6.

12.- Leoni F, Amar C, Nichols G, Pedraza-Díaz S, McLauchlin J. Genetic analysis of Cryptosporidium from 2414 humans with diarrhoea in England between 1985 and 2000. J Med Microbiol 2006; 55: 703-7.

13.- Palmer C J, Xiao L, Terashima A, Guerra H, Gotuzzo E, Saldías G, et al. Cryptosporidium muris, a rodent pathogen, recovered from a human in Perú. Emerg Infect Dis 2003; 9: 1174-6.

14.- Chalmers R M, Elwin K, Thomas A L, Joynson D H. Infection with unusual types of Cryptosporidium is not restricted to immunocompromised patients, J Infect Dis 2002; 185: 270-1.

15.- Mallon E, MacLeod A, Wastling J M, Smith H, Tait A. Multilocus genotyping of Cryptosporidium parvum type 2: population genetics and sub-structuring. Infect Genet Evol 2003; 3: 207-18.

16.- Chalmers R M, Robinson G, Elwin K, Hadfield S J, Xiao L, Ryan U, et al. Cryptosporidium sp. rabbit genotype, a newly identified human pathogen. Emerg Infect Dis 2009; 15: 829-30.

17.- Xiao L, Hlavsa M C, Yoder J, Ewers C, Dearen T, Yang W, et al. Subtype analysis of Cryptosporidium specimens from sporadic cases in Colorado, Idaho, New Mexico, and Iowa in 2007: widespread occurrence of one Cryptosporidium hominis subtype and case history of an infection with the Cryptosporidium horse genotype. J Clin Microbiol 2009; 47: 3017-20.

18.- Neira P, Muñoz N, Stanley B, Gosh M, Rosales M J. Cryptosporidium parvum en gastrópodos silvestres como bioindicadores de contaminación fecal en ecosistemas terrestres. Rev Chil Infectol 2010; 27: 211-8.

19.- Xiao L, Escalante L, Yang C, Sulaiman I, Escalante A A, Montali R, et al. Phylogenetic analysis of Cryptosporidium parasites based on the small subunit rRNA gene locus. Appl Environ Microbiol 1999; 65: 1578-83.

20.- Xiao L, Morgan UM, Limor J, Escalante A, Arrowood M, Shulaw W, et al. Genetic diversity within Cryptosporidium parvum and related Cryptosporidium species. Appl Environ Microbiol 1999; 65: 3386-91.

21.- Tiangtip R, Jongwutiwes S. Molecular analysis of Cryptosporidium species isolated from HIVinfected patients in Thailand. Trop Med Intern Health 2002; 7: 357-64.

22.- Neira-Otero P, Muñoz-Saldías N, SánchezMoreno M, Rosales-Lombardo MJ. Molecular characterization of Cryptosporidium species and genotypes in Chile. Parasitol Res 2005; 97: 63-7.

23.- Neira P, Muñoz N, Rosales M J. Infección por Cryptosporidium parvum en una mujer embarazada, inmunocompetente, con riesgo ocupacional. Rev Chilena Infectol 2010; 27 (4): 345-9.

24.- Neira P, VillalónL, Subercaseaux B. Cryptosporidium sp. Coccidio parásito de interés médico. Situación en la Va Región, Chile. Rev Méd Valparaíso 1989; 42: 51-6.

25.- Brantley R, Williams R, Silva T, Sistrom M, Thielman NM, Ward H, et al. AIDS-associated diarrhea and wasting in northeast Brazil is associated with subtherapeutic plasma levels of antiretroviral medications and with both bovine and human subtypes of Cryptosporidium parvum. Braz J Infect Dis 2003; 7:16-22.

26.- Flanigan T, Whalen C, Turner J, Soave R, Toerner J, Havlir D, et al. Cryptosporidium infection and CD4 counts. Ann Intern Med 1992; 116:840-2.

27.- Brink AK, Mahé C, Watera C, Lugada E, Gilks C, Whitworth J, et al. Diarrhea, CD4 counts and enteric infections in a community based cohort of HIV infected adults in Uganda. J Infect 2002; 45: 99-106.

28. Muthusamy D, Rao SS, Ramani S, Bindhu M, Banerjee I, Abraham OC, et al. Multilocus genotyping of Cryptosporidium sp. isolates from human immunodeficiency virus-infected individuals in South India. J Clin Microbiol 2006; 44: 632-4.

29.- Vajpayee M, Kanswal S, Seth P, Wig N. Spectrum of opportunistic infections and profile of CD4 counts among AIDS patients in North India. Infection 2003; 31: 336-40.

30.- Fayer R, Gasbarre L, Pasquali P, Canals A, Almeria S, Zarlenga D. Cryptosporidium parvum infection in bovine neonates: dynamic clinical, parasitic and immunologic patterns. Int J Parasitol 1998; 28: 49-56.

31.- Zintl A, Proctor A F, Read C, Dewaal T, Shanaghy N, Fanning S, et al. The prevalence of Cryptosporidium species and subtypes in human faecal samples in Ireland. Epidemiol Infect 2009; 137: 270-7.

32.- Feltus D C, Giddings C W, Schneck B L, Monson T, Warshauer D, McEvoy J M. Evidence supporting zoonotic transmission of Cryptosporidium spp in Wisconsin. J Clin Microbiol 2006; 44, 4303-8.

33.- Gatei W, Greensill J, Ashford R W, Cuevas L E, Parry C M, Cunliffe NA, et al. Molecular analysis of the 18S rRNA gene of Cryptosporidium parasites from patients with or without human immunodeficiency virus infections living in Kenya, Malawi, Brazil, the 
United Kingdom, and Vietnam. J Clin Microbiol 2003; 41: 1458-62.

34.- Cama V A, Ross J M, Crawford S, Kawai V, Chávez-Valdez R, Vargas D, et al. Differences in clinical manifestations among Cryptosporidium species and subtypes in HIV-infected persons. J Infect Dis 2007; 196: 684-91.

35.- Thompson R C, Olson M E, Zhu G, Enomoto S, Abrahamsen M S, Hijjawi N S. Cryptosporidium and cryptosporidiosis. Adv Parasitol 2005; 59: 77-158.

36.- Xiao L, Morgan U M, Fayer R, Thompson R C, Lal AA. Cryptosporidium systematics and implications for public health. Parasitol Today 2000; 16: 287-92.

37.- Sönmez Tamer G, Balikçi E, Erbay A. The prevalence of cryptosporidiosis in children who were diagnosed with leukemia and lymphoma. Turkiye Parazitol Derg 2008, 32 (3): 192-7.

38.- Prado V, Brinck M, Martínez D. Enteritis por Cryptosporidium sp en un paciente leucémico. Rev Chil Pediatr 1985, 56: 251-3.

39.- Roncoroni A J, Gómez M A, Mera J, Cagnoni P, Michel M D. Cryptosporidium infection in renal transplant patients. J Infect Dis 1989; 160: 559.

40.- Holley H P Jr, Thiers B H. Cryptosporidiosis in a patient receiving immunosupressive therapy. Possible activation of latent infection. Dig Dis Sci 1986; 31:1004-7.

41.- Chieffi P P, Sens Y A, Paschoalotti M A, Miorin L A, Silva H G, Jabur P. Infection by Cryptosporidium parvum in renal patients submitted to renal transplant or hemodialysis.
Rev Soc Bras Med Trop 1998; 31: 333-7.

42.- Ok U Z, Cirit M, Uner A, Ok E, Akcicek F, Basci A, et al. Cryptosporidiosis and blastocystosis in renal transplant recipients. Nephron 1997; 75: 171-4.

43.- Rehg JE, Hancock ML, Woodmansee DB. Characterization of cyclophosphamide-rat model of cryptosporidiosis. Infect Immun 1987; 55: 2669-74.

44.- Rehg J E, Hancock M L, Woodmansee D B. Characterization of dexamethasone-treated model of cryptosporidial infection. J Infect Dis 1988; 158: 1406-7.

45. McLauchlin J, Amar C F, Pedraza-Díaz S, Mieli-Vergani G, Hadzic N, Davies EG. Polymerase chain reaction-based diagnosis of infection with Cryptosporidium in children with primary immunodeficiencies. Pediatr Infect Dis J 2003; 22 (4): 329-35.

46.- Wolska-Kusnierz B, Bajer A, Caccio S, Heropolitanska-Pliszka E, Bernatowska E, Socha P, et al. Cryptosporidium infection in patients with primary immunodeficiencies. J Pediatr Gastroenterol Nutr 2007; 45: 458-64.

47.- Hayward A R, Levy J, Facchetti F, Notarangelo L, Ochs H D, Etzioni A, et al. Cholangiopathy and tumors of the pancreas, liver, and biliary tree in boys with X-linked immunodeficiency with hyper-IgM. J Immunol 1997; 158: 977-83.

48.- Hoveyda F, Davies W A R, Hunter P R. A case of cryptosporidiosis in pregnancy. Eur J Clin Microbiol Infect Dis 2002; 21: 637-8.

49.- Neira P, Villalón L. Cryptosporidiosis en la V Región, Chile. I. Estudio en pacientes con síndromes diarreicos, 1985-1987. Parasitol al día 1988; 12: 25-9.

50.- Araújo A J U S, Kanamura H Y, Almeida M E, Gomes A H S, Pinto THL, Da Silva A J. Genotypic identification of Cryptosporidium spp. isolated from HIV-infected patients and immunocompetent children of São Paulo, Brazil. Rev Inst Med trop S Paulo 2008; 50: 139-43.

51.- Abe N. Genotype analysis of Cryptosporidium meleagridis isolates from humans in Japan. Jpn J Infect Dis 2010; 63: 214-5.

52.- McLauchlin J, Amar C, Pedraza-Díaz S, Nichols G L. Molecular epidemiological analysis of Cryptosporidium spp. in the United Kingdom: results of genotyping Cryptosporidium spp. in 1,705 fecal samples from humans and 105 fecal samples from livestock animals. J Clin Microbiol 2000; 38; 3984-90.

53.- Pedraza-Díaz S, Amar C, Nichols G L, McLauchlin J. Nested polymerase chain reaction for amplification of the Cryptosporidium oocyst wall protein gene. Emerg Infect Dis 2001; 7: 49-56.

54.- Cama V A, Bern C, Sulaiman I M, Gilman R H, Ticona E, Vivar A, et al. Cryptosporidium species and genotypes in HIV-positive patients in Lima, Perú. J Eukaryot Microbiol 2003; 50: 531-3.

55.- Vidal S, Toloza L, Cancino B. Evolución de la prevalencia de enteroparasitosis en la ciudad de Talca, Región del Maule, Chile. Rev Chil Infectol 2010; 27: 336-40. 